\title{
Reflets discursifs des élites intellectuelles et culture classique à l'époque contemporaine
} Modes d'approches différenciés de l'espace grec

Reflection about the Relationships between Intellectual Elites and Ancient Greek Culture, in Particular in the Contemporary Greek Era

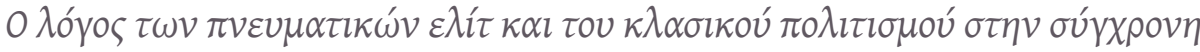

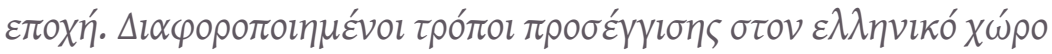

\section{Constantin Bobas}

\section{OpenEdition}

\section{Journals}

Édition électronique

URL : https://journals.openedition.org/ceb/5475

DOI : $10.4000 /$ ceb. 5475

ISSN : 2261-4184

Éditeur

INALCO

\section{Édition imprimée}

Date de publication : 1 mars 2015

ISBN : 978-2-85831-224-5

ISSN : 0290-7402

\section{Référence électronique}

Constantin Bobas, «Reflets discursifs des élites intellectuelles et culture classique à l'époque contemporaine », Cahiers balkaniques [En ligne], Hors-série | 2015, mis en ligne le 10 décembre 2015, consulté le 06 juillet 2021. URL : http://journals.openedition.org/ceb/5475 ; DOI : https://doi.org/ $10.4000 /$ ceb.5475

Ce document a été généré automatiquement le 6 juillet 2021

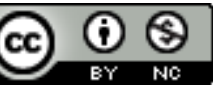

Cahiers balkaniques est mis à disposition selon les termes de la Licence Creative Commons Attribution - Pas d'Utilisation Commerciale 4.0 International. 


\title{
Reflets discursifs des élites intellectuelles et culture classique à l'époque contemporaine
}

\author{
Modes d'approches différenciés de l'espace grec \\ Reflection about the Relationships between Intellectual Elites and Ancient Greek \\ Culture, in Particular in the Contemporary Greek Era

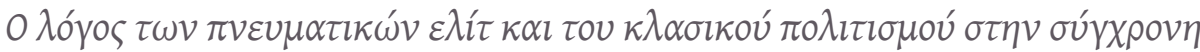

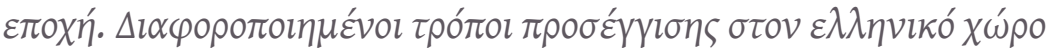

Constantin Bobas

La culture grecque classique a souvent été associée à la formation des élites intellectuelles, pas toujours à bon escient, en évoquant non seulement la période historique où elle a évolué, mais aussi l'époque nommée moderne, en tant que modèle et fondement pour un certain nombre de réalisations de la civilisation occidentale. À cette considération en quelque sorte inévitable pourrait venir se joindre cette homonymie qui rapproche la Grèce comme pays, et le terme moderne sous son acception temporelle, dans le mouvement de la création d'un État pour laquelle cette culture ancienne a joué incontestablement un rôle. La question est vaste, largement débattue et non dépourvue d'enjeux idéologiques, politiques et sociaux d'une équation d'autant plus complexe que l'évolution de ces rapports dépend de plusieurs paramètres modifiables. Même si l'effort déployé est invariablement le même, à savoir l'exigence d'atteindre le plus profondément possible la réalité de cette culture classique ainsi que la recherche dans certains cas d'un état fusionnel avec ses créations qui pourrait conduire à une plus grande connaissance de l'humain et à la perfectibilité de la société. Cependant, l'accès à cette culture ne donnera pas toujours satisfaction, surtout pendant des périodes de crise multiples et de questionnements inquiets sur l'avenir, provoquant une réflexion de la part des élites intellectuelles afin de redéfinir ses relations possibles avec le temps présent. Plus particulièrement, pour le monde néo-hellénique, cette réflexion, surtout dans la première moitié $\mathrm{du} \mathrm{xx}^{\mathrm{e}}$ siècle, a pris une dimension 
constitutive à la formation d'une nouvelle vision collective, grâce surtout aux représentants de la génération des années 1930, qui a marqué longtemps cette société ${ }^{1}$. La démarche engagée dans cette direction est due principalement à des conditions historiques, politiques et sociales internes à la Grèce, mais elle pourrait s'inscrire aussi dans un contexte général d'une recherche intellectuelle en Occident pour revenir aux sources de la culture grecque antique.

\section{Temps et mouvements, tours et détours pour une culture grecque ancienne}

2 Si la redécouverte de l'Antiquité grecque passe par la position nodale de la Renaissance italienne après la chute de Constantinople en 1453, sa réception semble parfois avoir choisi des voies spécifiques, et même paradoxales à première vue, notamment à l'époque contemporaine :

Ainsi, la situation de l'histoire du monde que l'on appelle, suivant la chronologie historique, l'époque moderne, est également fondée sur l'événement de la romanisation du grec. La « Renaissance » de l'Antiquité qui accompagne le début de l'époque moderne en est la preuve manifeste. Le fait qu'aujourd'hui encore nous voyions le monde grec avec des yeux romains, et cela non seulement dans les recherches des historiens du monde grec, mais, ce qui seul est décisif, dans l'explication métaphysique historique du monde moderne avec l'Antiquité, est une conséquence plus lointaine, mais nullement indifférente, de la romanisation du grec et de la renaissance romaine de l'Antiquité. [...] Dès l'instant où nous dirigeons notre regard vers les domaines d'essence simple qui, sans fracas et sans bruit, passent inaperçus aux yeux de l'historien, mais sont pourtant incontournables, alors, mais alors seulement, nous voyons nos représentations fondamentales habituelles, à savoir romaines, chrétiennes et modernes, dans leur indigence se briser au contact de l'essence initiale du grec.

Dans cet extrait, Heidegger (2011, 75-76) développe l'idée d'une perception romaine, latine, du monde grec ancien à la Renaissance ${ }^{2}$ qui perdurerait jusqu'à notre époque, même si cela correspond à une réalité historique déjà présente durant l'Antiquité où l'Empire romain évolue dans le cadre d'une culture principalement gréco-romaine ${ }^{3}$. Il y aurait donc "une essence initiale du grec " qui nous a échappé et continuera à nous échapper à partir du moment où une forme d'appréhension directe de cette civilisation, une autre approche heuristique ne se met pas en place pour découvrir sa véritable nature. Les raisons historiques qui ont conduit à la constitution de la culture renaissante, notion qui englobe des réalités diverses en Italie et en Europe, sont évidemment à l'origine, au moins d'un point de vue intellectuel, de cette perception et de l'influence exercée par une lecture réductrice de la tradition grecque antique.

4 En effet, cette culture, pratiquement disparue en Occident, continue toujours son mouvement dans l'Orient byzantin sous une autre forme, principalement christianisée, tout en perpétuant la tradition hellénistique, nourrie par une éducation classique. Une certaine animosité se ressentait entre les villes italiennes et Byzance autour de la connaissance de l'Antiquité grecque dès le xIve siècle, les villes italiennes considérant que la culture byzantine, malgré son prestige rattaché au monde grec, n'était qu'un avatar substantiellement inférieur de la Grèce antique. C'est probablement dans cette appréciation que la Grèce ancienne a été saisie, fondée aussi sur une certaine romanité grecque et s'opposant à la maniera greca byzantine, sans pour autant éviter 
complètement sa présence, surtout au travers de l'art et la transmission des connaissances par les artistes (CHASTEL, 1999, 84-88) et les érudits byzantins (SALADIN, 2004, 41-76). À cela, il faudrait ajouter que dans chaque pays où à cette période, ont eu lieu ces changements importants qui n'évoluent pas de la même façon partout, l'apport de la culture humaniste s'intègre différemment sous l'effet d'une interaction permanente. Et dans ce que nous appelons l'espace grec, entité spatiale très fluctuante selon les temporalités historiques, ce même mouvement ne peut exister que de manière très singulière, étant donné que les conditions ne sont pas réunies pour produire un contexte renaissant équivalent. Il serait très difficile d'évoquer par exemple la redécouverte de la culture grecque dans une civilisation où elle n'a jamais été totalement absente, même si elle évolue généralement sur des modes distincts. Par ailleurs, en raison de l'occupation ottomane, cette perspective ainsi que le développement des autres aspects principaux de la Renaissance, ne pouvait se poursuivre qu'à l'extérieur de cet espace ou bien dans des régions où l'environnement culturel le permettait, telles la Crète et plus tard, les îles Ioniennes.

Mais des mutations qui vont transformer encore profondément les sociétés européennes auront lieu au siècle des Lumières, fondées principalement sur la notion de progrès et d'esprit critique. Dans ce contexte général, il est possible d'observer des situations en apparence discordantes : l'adhésion à la culture grecque antique, mais le scepticisme concernant le classicisme du siècle précédent, l'acceptation d'un modèle humaniste, mais l'opposition au système éducatif qui s'en inspire. Les Lumières ne font que prolonger intensément l'engagement de la Renaissance en faveur de la raison contre une perception transcendante du monde. La pensée théologique, scolastique, sera pleinement contestée, souvent par l'expression d'un certain agnosticisme qui mettra les possibilités humaines au centre de la vie, en essayant d'écarter Dieu, la référence absolue du christianisme, abordé par le biais de la métaphysique aristotélicienne ou platonicienne.

\section{Éléments conjoncturels de la culture grecque antique dans l'espace néo-hellénique}

6 Ainsi, l'héritage grec ancien auquel l'on considère qu'une partie de la culture de l'Europe doit son développement, par des oscillations fréquentes, exerce une grande influence dans l'imaginaire, mais aussi dans la réalité des mouvements intellectuels de l'époque. Mais il s'agit d'un monde grec qui continue à correspondre à une modalité plutôt utopique de l'Occident, avant de (re)découvrir progressivement que, selon toute vraisemblance, il existe aussi une réalité physique d'un territoire où ses habitants parlent une langue grecque et vivent dans cet espace occupé alors par l'Empire ottoman. Cette conjoncture se prêtant à des réflexions sur les rapports de l'ancien et du moderne offre l'occasion à l'élite intellectuelle de ces populations, vivant surtout à l'extérieur de cet espace, dans un contexte idéologique approprié, de revendiquer l'avènement d'une autre configuration possible pour un meilleur avenir. Or, des efforts ont été déployés afin de projeter la vision de la constitution d'un État-Nation en l'inscrivant, entre autres, dans le champ culturel de l'hellénisme, pensé également par les grandes puissances européennes. Le mouvement influent du philhellénisme évolue dans cette même direction en tant que phénomène unique, vers la fin de cette période en Europe, combinant des caractéristiques aussi bien politiques et idéologiques que 
culturelles et artistiques. Il contribue ainsi à la lutte des Grecs pour leur libération des Ottomans en soutenant leur action pour l'indépendance, revêtant différentes formes d'argumentation, l'Europe contre l'Asie, le christianisme contre l'islam... (JUSDANIS, 1991, 13-17).

7 C'est dans ce contexte aussi que les idées des Lumières seront intégrées dans la problématique des penseurs grecs, même si cela ne se fait pas sans heurt. Coray craint que si les Grecs n'adoptent pas les valeurs des Lumières et restent attachés aux "moines", les Européens ne suivent une politique pro-ottomane. Tandis qu'en y adhérant, cette tendance serait inversée et la Grèce trouverait sa place dans le concert des nations (KONDYLIS, 1998, 204-205). En effet, comme dans la période qui précède la fin de l'Empire byzantin, au moment où les fondements de la pensée de la Renaissance apparaissent, surviennent des mouvements qui considèrent différemment la tradition grecque $^{4}$. De manière presque équivalente, dans son sillage, une autre réaction se manifeste sous la forme de la pensée des moines du mont Athos, contre les idées des Lumières ${ }^{5}$. Une telle situation aura souvent pour corollaire une ambiguïté, une position parfois équivoque de certains représentants des Lumières grecques concernant leurs rapports avec la religion, annonciatrice des difficultés inhérentes à une sécularisation de la société qui ne suivra pas les mêmes processus que dans les autres pays européens.

Cependant, cette forme de polarisation, apparemment inconciliable, entre une représentation sacrée du monde et une approche profane, n'a pas empêché le discours idéologique contemporain à la fondation de l'État néo-hellénique, de faire appel à la culture de l'Antiquité grecque, le point de jonction majeur avec les pays occidentaux.

\section{Les élites intellectuelles néo-helléniques et l'Antiquité à l'époque contemporaine : variantes et constructions}

9 Suivant les époques, les sensibilités ambiantes, les rapports interculturels, les préoccupations politiques et sociales, cette évocation de l'Antiquité a revêtu des sens divers exprimés par les élites intellectuelles dans le cadre de la société néo-hellénique. Elle oscille entre la revendication pure et simple d'une descendance évidente et la distance réfléchie en tant que conséquence de la succession de civilisations historiquement éloignées et culturellement différentes. Parmi ces approches, certaines sont directement liées à une image de la Grèce venant de l'extérieur, d'un point de vue occidental, et d'autres associant des éléments extérieurs, occidentaux, avec la quête d'une tradition spécifique, tantôt gréco-byzantine, tantôt d'inspiration populaire. Une autre possibilité se présente à travers une expression singulière où toute intervention qui ne s'y greffe pas naturellement est rejetée, qu'il s'agisse d'une perspective antique ou populaire. D'une manière plus précise, concernant l'approche de l'Antiquité grecque dans le monde néo-hellénique aux $\mathrm{xIx}^{\mathrm{e}}$ et $\mathrm{xx}^{\mathrm{e}}$ siècles, il est possible d'observer des expressions qui évoluent dans un cadre symbolique/archéologique, surtout lors des premières décennies de l'existence de l'État grec; des démarches qui passent par une dimension organique/romantique; celles pouvant être qualifiées d'ironiques en prenant une certaine distance critique; et enfin, celles d'une élaboration esthétique fonctionnant de manière archétypique (TzIOVAS, 2011, 306-320, et 2008, 287-298). Pour ce dernier cas qui constitue une proposition intégrale, en particulier de la part de la génération des années 1930, dont l'incidence reste encore significative, Georges Séféris 
distingue bien au sein de cette culture classique l'hellénisme européen de l'hellénisme grec $(1987,35-37)$ :

[...] L’hellénisme s'est trouvé modelé, pétri, vivifié par des courants parfois grecs, parfois non, et ceci jusqu'à la Renaissance. À partir de cette époque, qui fut celle de l'asservissement de la nation, les forces agissantes ne furent plus grecques et se situèrent hors de l'espace hellénique. C'est à partir de cette époque, ne l'oublions pas, que virent le jour les cuvres en lesquelles nous reconnaissons les caractéristiques de ce que nous appelons aujourd'hui la culture occidentale. [...]. L'Ulysse de Dante, Vénus et Adonis de Shakespeare, la Phèdre de Racine, l'Hypérion de Hölderlin sont tous, indépendamment de leur valeur artistique générale, des figures ayant une profonde appartenance à l'époque et au peuple de leurs créateurs, et l'hellénisme ne constitue chez elles qu'un prétexte superficiel.

Seulement voilà : animés de fort louables intentions, enflammés du désir de faire revenir chez nous tout ce qui est grec, nous nous sommes laissés prendre à des dehors grecs pour charrier en Grèce mille valeurs étrangères, sans penser qu'elles n'avaient aucun rapport réel avec notre patrie. [...].

J'ai dit que l'hellénisme est difficile. En effet, si, dans la vie de l'esprit, il existe bien un hellénisme européen et qui est peut-être aujourd'hui sur le point d'expirer, « l'hellénisme grec »-qu'on me permette cette expression - n'est pas encore né et manque d'une tradition établie. [...].

Si j'ai raison, le problème se résume donc au degré de vérité et de profondeur du regard que le Grec portera et sur lui-même et sur sa nature, partie intégrante de la grande nature grecque ${ }^{6}$.

Ce texte écrit en 1938 évoquant la dissemblance entre l'« hellénisme européen » et l'« hellénisme hellénique ", résonne étrangement ${ }^{7}$ avec celui de Heidegger composé quasiment à la même époque. Dans les deux cas, est exprimée la nécessité d'entreprendre une autre approche de la culture grecque, mais avec des objectifs et des moyens contrastés. Soulignons ici que la différenciation concernant l'Antiquité classique correspond, pour le monde occidental surtout, à une appréhension de cette culture afin de l'intégrer le plus souvent dans un contexte d'érudition, considérée dans le cadre de l'éducation humaniste comme une valeur absolue, synonyme d'un statut social. Pourtant, il faudrait signaler que, dans les sociétés occidentales, dès les dernières décennies $d u \mathrm{XIX}^{\mathrm{e}}$ siècle, des évolutions sont déjà perceptibles, mettant en cause cette disposition dont l'ascendant commence à perdre de l'importance. Elle sera encore présente pendant une certaine période, mais au profit d'expressions qui visent à une production culturelle fondée sur l'adhésion du plus grand nombre, en insistant sur l'obligation pour des utilisations efficientes. Ainsi, progressivement, la place de l'homme au centre de la société prend une dimension de masse, notamment à partir du $\mathrm{xx}^{\mathrm{e}}$ siècle, un changement majeur depuis la Renaissance et les Lumières où l'essence humaine, sous la forme de l'homme-créateur, s'organise en étendue humaine qui domine désormais son rapport à la réalité matérielle de la vie (FoucAuLT, 1966, 314-354 et Deleuze, 1990, 136-137). Cet état d'agitation sociale et de crise caractéristique de la période où un monde ancien est en train de disparaître de par les aspirations d'un mouvement multiforme, introduit une nouvelle conception de la société, de facture populaire face aux élites traditionnelles. Il est, sans doute, révélateur de ce point de vue que la civilisation européenne qui s'était imposée comme culture dominante, perde au $\mathrm{Xx}^{\mathrm{e}}$ siècle sa position prépondérante au profit justement de manifestations culturelles de masse et avant que la suprématie occidentale, dans la continuité temporelle, ne cède la place à des expressions venant de multiples horizons. 
11 La remarque de Séféris sur l'« hellénisme hellénique ${ }^{8}$ » qui n'existe pas encore et est à créer, malgré toutes les difficultés inhérentes, désigne l'élaboration d'un autre schéma conceptuel de la culture néo-hellénique. Il est fondé sur ses rapports aussi bien avec l'Antiquité qu'avec la civilisation européenne, tout en s'inscrivant dans les mouvements modernistes de son époque sur le fonctionnement de la tradition classique ${ }^{9}$. Dans cette perspective, la singularité fondatrice de la Grèce peut être à nouveau révélée où la construction de sa physionomie, pour les autres pays, notamment européens, passe par une confrontation permanente avec un miroitement antique et sa comparaison avec la culture classique, positivement ou négativement, selon les avatars de sa propre trajectoire et de l'histoire commune. À cette perception, ses élites intellectuelles se sentent obligées de répondre, soit en considérant que constitue une nécessité vitale pour l'existence du pays l'inclusion d'un autre rapport avec l'Antiquité dans la construction des nouveaux mythes fondateurs (TZIOVAS, 2011, 152-154), soit à cause d'une aspiration forte de reconnaissance, ou bien les deux à la fois. En tout cas, ce qui en résulte est une difficulté durable pour que la Grèce puisse se définir autrement que d'après une représentation échafaudée qui lui est envoyée de l'extérieur en ignorant et parfois en rejetant sa propre évolution historique. Sa constitution moderne renvoie principalement à des valeurs culturelles sur fond d'éléments géopolitiques, économiques, politiques et sociaux autrement plus importants qui produisent souvent une certaine incompatibilité d'objectifs. Ce déséquilibre érigé, parfois, en dispositif de survie sert et est servi par ces élites, dans toute circonstance critique, pour défendre des intérêts collectifs, mais qui ont justifié dans un certain nombre de cas des manquements ou des interventions abusives venant, souvent, de l'extérieur.

\section{Lectures croisées. Une autre (post)-modernité possible de la culture grecque?}

Les approches de Heidegger et de Séféris, malgré leurs points communs dus, sans doute, à un contexte discursif équivalent, reflètent aussi des différences correspondant à une vision divergente du monde grec classique dont les fondements sous-tendent des facteurs externes et internes dans ce processus de réappropriation réflexive. En effet, la démarche de Heidegger, provenant d'une approche occidentale, met en cause la romanisation du grec afin d'incorporer sa vraie essence dans un contexte anhistorique intégrant, seul capable de permettre un dialogue synchronique entre les hommes et l'expérience d'une relation de vérité commune. Séféris, dans son rapprochement évident, met en cause l'introduction de valeurs, grecques en apparence seulement, mais qui sont étrangères à la situation du pays, en insistant sur une perspective historicoorganique qui doit être adoptée dans l'objectif de créer sa propre tradition, pas encore advenue, en phase avec sa propre vérité. Mais, le présent perpétuel de Heidegger dans sa conception anhistorique pourrait s'articuler, dans une certaine mesure, avec la mémoire historique de Séféris qui se focalise sur l'espace, l'esthétisation et l'humanisation d'un territoire dans sa réalité diachronique. Or, il ne s'agirait que de séquences complémentaires à la construction d'une multiplicité conceptuelle en devenir de la culture grecque.

13 Cependant, une observation rétroactive de ces développements théoriques parallèles et leur incidence contemporaine mettrait en évidence une certaine inadéquation entre les réalisations intellectuelles et les événements culturels, entre les principes directeurs 
d'une élite intellectuelle et les évolutions culturelles et sociales. Les approches, essentialiste de Heidegger et d'esthétisation territoriale de Séféris, correspondaient bien à leur époque, et s'intègrent complètement dans leurs œuvres, mais elles n'ont pas trouvé de véritable continuation en restant en suspens, ou bien n'ont pas produit pleinement les résultats escomptés. Sans doute, parce qu'elles considéraient la culture classique comme une référence principale, prédominante, sur laquelle une ou plusieurs traditions pourraient venir s'attacher en établissant un nouveau schéma de pensée. Une telle appréhension ressemble à une définition de la modernité, au sens baudelairien du terme : «La modernité, c'est le transitoire, le fugitif, le contingent, la moitié de l'art, dont l'autre moitié est l'éternel et l'immuable. Il y a eu une modernité pour chaque peintre ancien " (BAUDELAIRE, 1951, 884). Les combinaisons possibles entre ces deux perspectives - qui peuvent être désignées par les termes de tradition active et de modernité - sont innombrables, oscillant entre collusion salutaire et conflit violent.

Cette configuration pourrait expliquer aussi, dans un cadre conceptuel général, la formation même des élites, si elle est observée à travers le fonctionnement hiérarchique de différentes composantes au sein d'une société donnée. Mais, à l'époque de la postmodernité, de changement de paradigme que les deux auteurs avaient bien pressenti, il semblerait que la bipolarité individu-masse a été remplacée par des "dividuels ", des échantillons et des données (DELEUZE, 1990, 243-245), sans véritable hiérarchisation, qui sous diverses formes apparaissent aussi bien dans la société que dans la culture. Or, il est possible de penser que les deux éléments constitutifs de la définition de la modernité, dont l'un est stable et l'autre instable, se sont transformés dans notre "postmodernité » en deux éléments variables aux possibilités infinies de combinaison.

Dans cette perspective, en revenant sur la question des élites néo-helléniques, d'autres horizons peuvent se manifester actuellement où il n'y aurait plus de positions supérieures les unes aux autres, mais des potentialités de compositions à l'intérieur ou à l'extérieur de ces mondes grecs, entre civilisations, entre cultures, et dans l'hybridité de leur création.

La constatation a été faite du manque de construction, dès la fondation de l'État néo-

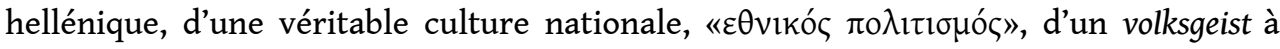
cause de l'existence d'une culture grecque classique qui s'impose de l'extérieur (TSOUKALAS, 2012, 37-38). Les autres pays européens, pour leurs propres raisons historiques, ont procédé à cette formation que les élites néo-helléniques ont souvent adoptée en intégrant cette partie de l'«éternel» et de l'«immuable» qui n'était devenue, entretemps, que du « fugitif » et du « contingent ». Cela a produit un discours, non seulement dans un contexte esthétique ou éthique, mais de manière récurrente, sur une énigme grecque moderne, l'«énigme de l'existence d'un peuple», les «miraculés de l'histoire »... Est-ce cela aussi la romanisation du grec dont parle Heidegger dans son versant grec moderne? Ou bien, la période actuelle qui affirme qu'écrire l'Histoire et écrire des histoires (RANCIÈRE, 2000, 32) n'est pas si différent, quelles qu'elles soient, en rapport avec le territoire, la culture ou la société ? C'est sans doute cette prise de conscience qui pourrait faire changer les choses dans un engagement nouveau, non plus dans un milieu élitiste, mais "élitaire pour tous " (VITEZ, 206, 12), capable de réinventer ou de ré-enchanter le monde, en considérant que c'est sur ce point que les différentes modalités d'approches pourraient se rencontrer. 


\section{BIBLIOGRAPHIE}

BAUDELAIRE Charles, 1951, « la Modernité », le Peintre de la vie moderne, in Yves-Gérard LE DANTEC (éd.), CEuvres complètes, Paris : Gallimard, coll. Bibliothèque de la Pléiade.

CHASTEL André, 1999, l'Italie et Byzance, Paris : Éditions de Fallois.

DELEUZE Gilles, 1990, Pourparlers, Paris : Éditions de Minuit.

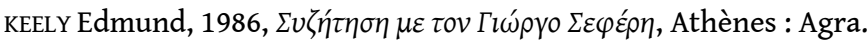

ELIot Thomas Stearns, 1985, “'Ulysses', Order and Myth”, in Frank Kermode (ed.), Selected Prose of T. S. Eliot, London: Faber and Faber.

FOUCAUlT Michel, 1966, les Mots et les Choses, Paris : Gallimard.

HEIDEGGER Martin, 2011, Parménide, Paris : Gallimard/Nrf.

JUSDANIS Gregory, 1991, Belated Modernity and Aesthetic Culture, Inventing National Literature, Minneapolis: University of Minnesota.

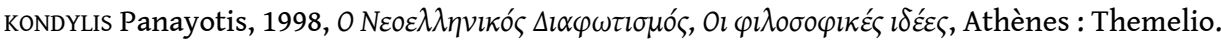

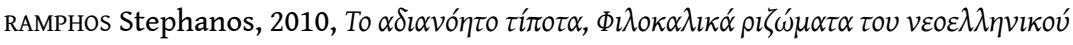

$\mu \eta \delta \varepsilon v l \sigma \mu o v ́$, Athènes : Armos.

RANCIÈRE Jacques, 2000, le Partage du sensible, Arles : la Fabrique.

SALADIN Jean-Christophe, (2004), la Bataille du grec à la Renaissance, Paris : les Belles Lettres.

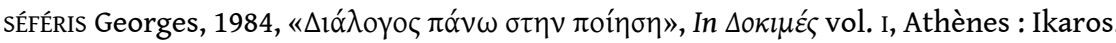

SÉFÉRIS Georges, 1987, « De la grécité en art » in Essais. Hellénisme et création. Traduction, choix et présentation D. KOHLER, Paris : Mercure de France.

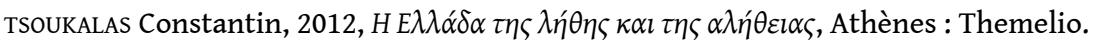

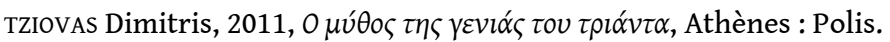

TZIOVAS Dimitris, 2008, "Reconfiguring the past: Antiquity and Greekness", in Dimitris DAMASKOS, Dimitri PLANTzos (eds), A singular Antiquity, Athènes: Musée Benaki.

VEYNE Paul, 2005, l'Empire gréco-romain, Paris : Seuil.

VITEZ Antoine, 2006, Antoine Vitez, Introduction et choix de textes N. LÉGER. Arles : Actes Sud.

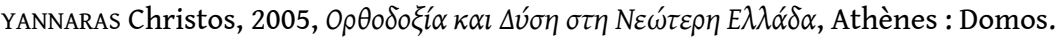

\section{NOTES}

1. Il ne s'agit pas ici de proposer un schéma culturaliste pour expliquer les orientations et l'évolution d'un État, mais la question de la culture ancienne dans le cas de la Grèce moderne est si intimement liée à sa structuration qu'il faudra la prendre en considération avec les autres facteurs fondamentaux dans sa constitution. 
2. L'ouvrage correspond aux cours sur Parménide donnés durant le semestre d'hiver 1942-1943 à l'Université de Fribourg-en-Brisgau, où cette question est abordée notamment au travers de la différence entre le mot grec $\alpha \lambda \eta^{\prime} \theta \varepsilon i \alpha$ et le mot latin veritas.

3. Paul VEYNE (2005) a particulièrement développé cette question de l'Empire romain en tant qu'Empire gréco-romain à l'origine de la culture européenne actuelle.

4. Dans une telle perspective pourrait s'inscrire, par exemple, la controverse de Grégoire Palamas et Barlaam le Calabrais au XIV siècle pendant la période de la querelle hésychaste.

5. Il s'agit du mouvement développé pendant la période de la composition du recueil anthologique de la Philocalie des Pères neptiques publié à Venise en 1782, lié à la spiritualité orthodoxe, une question qui a provoqué et provoque parfois encore des débats et des polémiques (YANNARAS, 2005, RAMPHOS, 2010).

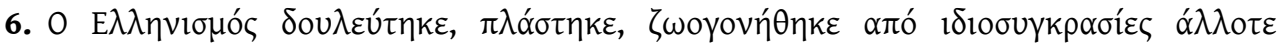

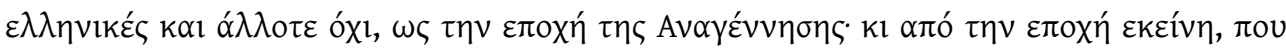

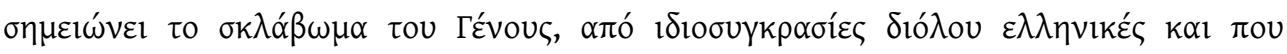

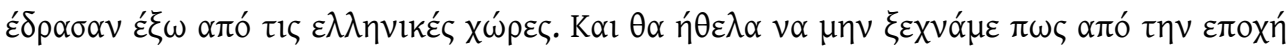

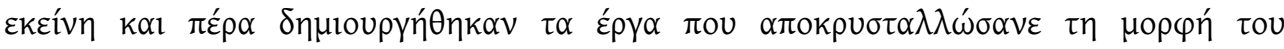

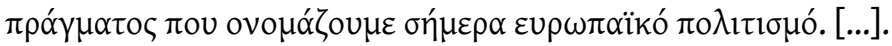

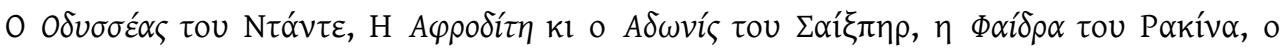

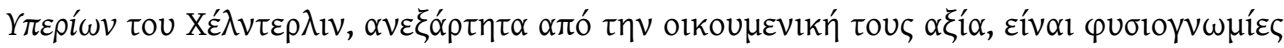

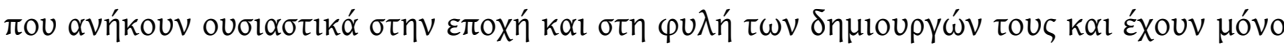

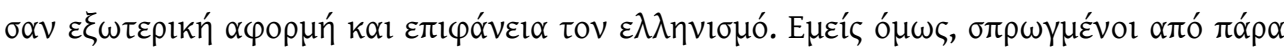

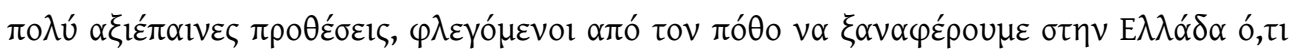

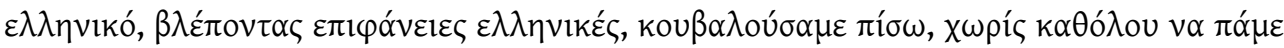

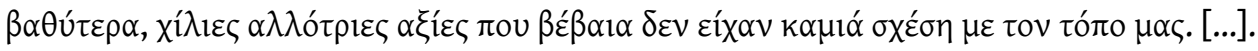

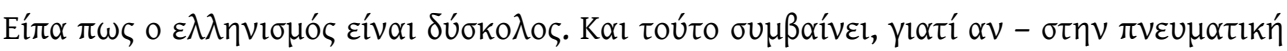

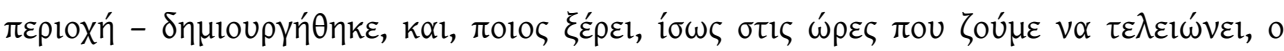

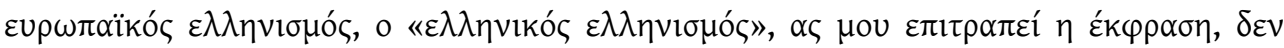

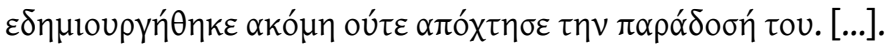

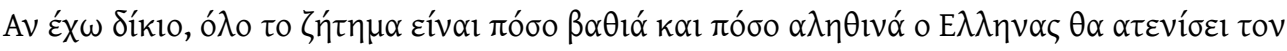

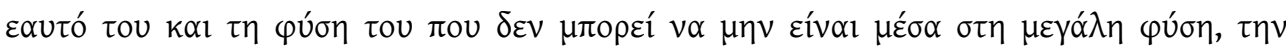
$\varepsilon \lambda \lambda \eta$ vikń. (Séféris, 1984, 99-102).

7. La référence à la notion de "vérité » $(\alpha \lambda \eta ́ \theta \varepsilon \varepsilon \alpha)$, en tant que modalité relative aux objectifs à atteindre, est à cet égard particulièrement révélatrice.

8. Plus tard, Séféris précisera le terme en le mettant en rapport avec la "grécité " ( $\Sigma \lambda \lambda \eta \nu ı \kappa o ́ \tau \eta \tau \alpha)$, et en lui donnant aussi d'autres significations (KEELY, 1986, 133).

9. Dans ce cadre, Séféris intègre aussi la «méthode mythique " qu'Eliot évoque à propos d'Ulysse de J. JOYCE $(1985,177)$. 


\section{RÉSUMÉS}

Cet article propose une réflexion sur les rapports que les élites intellectuelles ont entretenus avec la culture grecque antique, notamment à l'époque contemporaine. Tout en se concentrant sur l'espace néo-hellénique, il met en parallèle des approches venant d'autres horizons afin d'étudier certaines modalités de leur fonctionnement.

This paper proposes a reflection about the relationships between intellectual elites and ancient Greek culture, in particular in the contemporary era. While focusing on the Modern Greek cultural area it brings together approaches from other horizons in order to examine some of the processes by which these relationships function.

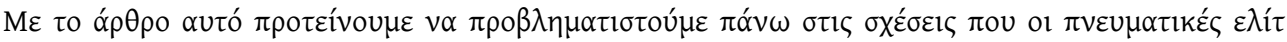

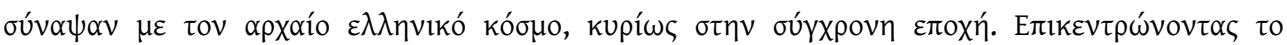

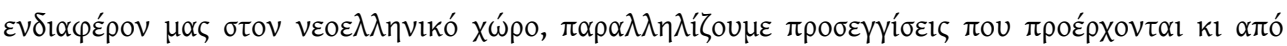

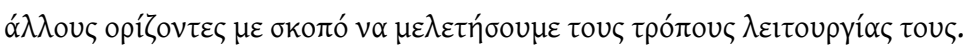

\section{INDEX}

Index géographique : Grèce

motsclestr Helenizm, Yunan kimliği, Yunanistan, Ondokuzuncu Yüzyıl

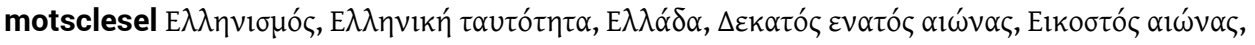

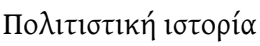

motsclesmk ХЕЛЕНИЗМОТ, ГРЧКИОТ ИДЕНТИТЕТ, ГРЦИЈА, ДЕВЕТНАЕСЕТТИОТ ВЕК, ДВАЕСЕТТИОТ ВЕК, КУЛТУРНАТА ИСТОРИЈА

Keywords : Hellenism, Greek identity, Greece, Nineteenth century, Twentieth century, Cultural history

Mots-clés : hellénisme, identité grecque

Thèmes : Histoire culturelle

Index chronologique : vingtième siècle, dix-neuvième siècle

\section{AUTEUR}

\section{CONSTANTIN BOBAS}

Université Charles de Gaulle - Lille 3 